\title{
Electric field modification of magnetotransport in Ni thin films on (011) PMN-PT piezosubstrates
}

Cite as: Appl. Phys. Lett. 106, 062404 (2015); https://doi.org/10.1063/1.4907775

Submitted: 22 December 2014 . Accepted: 28 January 2015 . Published Online: 10 February 2015

Alexander Tkach, Andreas Kehlberger, Felix Büttner, Gerhard Jakob, Stefan Eisebitt, and Mathias Kläui
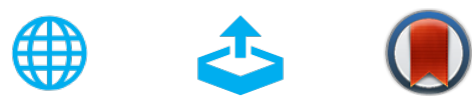

View Online

Export Citation

CrossMark

\section{ARTICLES YOU MAY BE INTERESTED IN}

Domain engineered switchable strain states in ferroelectric $\left.(011)\left[\mathrm{Pb}\left(\mathrm{Mg}_{1} /{ }_{3} \mathrm{Nb}_{2} / 3\right) \mathrm{O}_{3}\right]_{(1-\mathrm{x}}\right)^{-}$ $\left[\mathrm{PbTiO}_{3}\right]_{\mathrm{X}}(\mathrm{PMN}-\mathrm{PT}, \mathrm{x} \approx 0.32)$ single crystals

Journal of Applied Physics 109, 124101 (2011); https://doi.org/10.1063/1.3595670

Giant electric-field-induced reversible and permanent magnetization reorientation on magnetoelectric $\mathrm{Ni} /(011)\left[\mathrm{Pb}\left(\mathrm{Mg}_{1 / 3} \mathrm{Nb}_{2 / 3}\right) \mathrm{O}_{3}\right]_{(1-\mathrm{x})}-\left[\mathrm{PbTiO}_{3}\right]_{\mathrm{X}}$ heterostructure

Applied Physics Letters 98, 012504 (2011); https://doi.org/10.1063/1.3534788

Electrical switching of the magnetic vortex circulation in artificial multiferroic structure of Co/ Cu/PMN-PT(011)

Applied Physics Letters 110, 262405 (2017); https://doi.org/10.1063/1.4990987

\section{Lock-in Amplifiers up to $600 \mathrm{MHz}$}
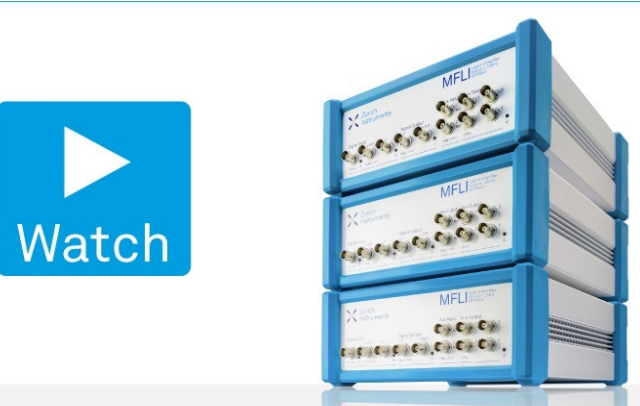


\title{
Electric field modification of magnetotransport in Ni thin films on (011) PMN-PT piezosubstrates
}

\author{
Alexander Tkach, ${ }^{1,2}$ Andreas Kehlberger, ${ }^{1,3}$ Felix Büttner, ${ }^{1,3,4}$ Gerhard Jakob, ${ }^{1,3}$ \\ Stefan Eisebitt, ${ }^{4}$ and Mathias Kläui ${ }^{1,3}$ \\ ${ }^{1}$ Institute of Physics, Johannes Gutenberg University, Staudinger Weg 7, 55128 Mainz, Germany \\ ${ }^{2}$ CICECO - Aveiro Institute of Materials, Department of Materials and Ceramic Engineering, University of \\ Aveiro, 3810-093 Aveiro, Portugal \\ ${ }^{3}$ Graduate School of Excellence Materials Science in Mainz, Staudinger Weg 9, 55128 Mainz, Germany \\ ${ }^{4}$ Institute of Optics and Atomic Physics, Technical University of Berlin, Str. des 17 Juni 135, 10623 Berlin, \\ Germany
}

(Received 22 December 2014; accepted 28 January 2015; published online 10 February 2015)

\begin{abstract}
This study reports the magnetotransport and magnetic properties of $20 \mathrm{~nm}$-thick polycrystalline $\mathrm{Ni}$ films deposited by magnetron sputtering on unpoled piezoelectric (011) $\left[\mathrm{PbMg}_{1 / 3} \mathrm{Nb}_{2 / 3} \mathrm{O}_{3}\right]_{0.68^{-}}$ $\left[\mathrm{PbTiO}_{3}\right]_{0.32}$ (PMN-PT) substrates. The longitudinal magnetoresistance (MR) of the Ni films on (011) PMN-PT, measured at room temperature in the magnetic field range of $-0.3 \mathrm{~T}<\mu_{0} \mathrm{H}<0.3 \mathrm{~T}$, is found to depend on the crystallographic direction and polarization state of piezosubstrate. Upon poling the PMN-PT substrate, which results in a transfer of strain to the Ni film, the MR value decreases by factor of 20 for the current along [100] of PMN-PT and slightly increases for the [01 $\overline{1}$ ] current direction. Simultaneously, a strong increase (decrease) in the field value, where the MR saturates, is observed for the [011] ([100]) current direction. The anisotropic magnetoresistance is also strongly affected by the remanent strain induced by the electric field pulses applied to the PMN-PT in the non-linear regime revealing a large $(132 \mathrm{mT})$ magnetic anisotropy field. Applying a critical electric field of $2.4 \mathrm{kV} / \mathrm{cm}$, the anisotropy field value changes back to the original value, opening a path to voltage-tuned magnetic field sensor or storage devices. This strain mediated voltage control of the MR and its dependence on the crystallographic direction is correlated with the results of magnetization reversal measurements. (C) 2015 AIP Publishing LLC.
\end{abstract}

[http://dx.doi.org/10.1063/1.4907775]

The conventional control of magnetization by currentgenerated magnetic fields is disadvantageous for electronic devices and sensors due to difficulties in reducing power consumption and realizing device miniaturization resulting from poor scaling. The non-volatile voltage control of the magnetization, resistivity or magnetoresistance (MR) in multiferroic heterostructures is one of the most promising schemes for achieving energy-efficient electronic applications. ${ }^{1}$ Such artificial multiferroics consist of ferro(i)magnetic and ferroelectric layers and parameters of the former one are controlled by a polarization of the latter one switched by external electric field with maximum energy efficiency. ${ }^{2}$ There are numerous reports on voltage control of magnetization (see, e.g., reviews, ${ }^{1-4}$ references therein, and recent Refs. 5-11), whereas for electric field effects on the anisotropic $^{12-20}$ and giant ${ }^{13,21,22} \mathrm{MR}$ in magnetic/ferroelectric heterostructures only few reports exist.

Recently, much attention has been focused on strainmediated magnetoelectric coupling between high- $\mathrm{T}_{\mathrm{c}}$ ferro(i)magnetic films such as $\mathrm{Ni}, \mathrm{Ni}-\mathrm{Fe}$, or $\mathrm{Fe}_{3} \mathrm{O}_{4}$ and (011)-oriented ferroelectric substrates with high room-temperature piezoelectric coefficients such as $\left[\mathrm{PbMg}_{1 / 3} \mathrm{Nb}_{2 / 3} \mathrm{O}_{3}\right]_{1-\mathrm{x}}-\left[\mathrm{PbTiO}_{3}\right]_{\mathrm{x}}$ $(\mathrm{PMN}-\mathrm{PT})$ or $\left[\mathrm{PbZn}_{1 / 3} \mathrm{Nb}_{2 / 3} \mathrm{O}_{3}\right]_{1-\mathrm{x}}-\left[\mathrm{PbTiO}_{3}\right]_{\mathrm{x}} \quad(\mathrm{PZN}-$ $\mathrm{PT}){ }^{4-11,13,14,16,17,20}$ In such structures, upon application of an electric field, the piezoactive substrate induces a strain in the ferro(i)magnetic film and hence modifies its magnetic properties due to the magnetoelastic coupling effect. The (011) cut is particularly suitable because of the possibility of obtaining a high and well-defined uniaxial anisotropy by inducing simultaneously compressive and tensile strains in orthogonal [100] (x) and $[01 \overline{1}](y)$ in-plane directions due to the different signs of $\mathrm{d}_{31}$ and $\mathrm{d}_{32}$ piezocoefficients. ${ }^{5,13}$ Voltage control of magnetization has been widely studied for Ni films on (011) PMN-PT using magneto-optic Kerr effect (MOKE) magnetometry and magnetic imaging, ${ }^{7-9,11}$ whereas the electric field effects on the MR have only been studied in detail for magnetite ${ }^{16,20}$ and permalloy films ${ }^{17}$ on (011) PMN-PT. Additionally, the MR response of permalloy films as a function of the electric field applied to the (011) PZN-PT piezosubstrate has been reported. ${ }^{13,14}$ Permalloy exhibits, however, a small magnetoelastic coupling constant, while its study on PMN-PT was made available only at finite magnetic fields. ${ }^{17}$ Moreover, to be useful for a device, one needs a change of the resistance at zero applied voltage, which has not been demonstrated using the application-relevant electrical readout. Furthermore, for 3dmetals, Ni exhibits the highest magnetoelastic coupling and is thus the material of choice where, by determining the full field dependence of the magnetotransport properties, one can gauge the applicability of this approach.

In this work, we report the results of magnetotransport characterization of $20 \mathrm{~nm}$-thick polycrystalline Ni films deposited by magnetron sputtering on unpoled (011)-oriented PMN-PT piezosubstrates. We determine the variation of the magnetotransport with the induced changes in the magnetic anisotropy resulting from the strain generated by PMN-PT poling and reversible and irreversible non-volatile switching 
by electric fields, and correlate the magnetoresistance changes with the magnetization manipulation.

The samples were prepared on polished (011)-oriented $\left[\mathrm{PbMg}_{1 / 3} \mathrm{Nb}_{2 / 3} \mathrm{O}_{3}\right]_{1-\mathrm{x}}-\left[\mathrm{PbTiO}_{3}\right]_{\mathrm{x}}(\mathrm{x}=0.32)$ substrates (Atom Optics Co., Ltd., Shanghai, China) of $5 \times 5 \times 0.5 \mathrm{~mm}^{3}$ size that were annealed at $300{ }^{\circ} \mathrm{C}$ for $30 \mathrm{~min}$. First, top $(10 \mathrm{~nm})$ and bottom $(50 \mathrm{~nm})$ Pt layers were DC-magnetron sputtered on both sides of the PMN-PT substrate using $5 \mathrm{~nm}$-thick adhesion layers of $\mathrm{Cr}$. Then, polycrystalline Ni films with a thickness of $20 \mathrm{~nm}$ were deposited on the top side by DC-magnetron spattering using an Ar pressure of $10^{-2}$ mbar.

Magnetoresistance measurements were performed in a van der Pauw configuration within a He cryostat (Oxford Instruments, MicrostatHe), using a system electrometer (Keithley 6514) as a current source and a 6.5-digit multimeter (Agilent 34411A) as a voltmeter with an error for our measurement range of $\sim 0.02 \%$. The magnetic field up to $0.3 \mathrm{~T}$ was generated by an electromagnet (GMW 3470) powered by a bipolar power supply (Kepco BOP 36-6M). A voltage supply $(\mathrm{FuG})$ was used for application of the electric fields up to $4 \mathrm{kV} / \mathrm{cm}$ between the top (Ni film) and bottom (Pt) electrodes of PMN-PT. The magnetization loops were obtained by a superconducting quantum interference device (SQUID) magnetometer (Quantum Design MPMSXL) along the $\boldsymbol{x}$ [100] and $\boldsymbol{y}$ [01 $\overline{1}$ ] directions with further subtraction of substrate diamagnetic component.

Figure 1 shows the room-temperature longitudinal magnetoresistance ratio $\Delta R / R_{0}=\left[R(H)-R_{0}\right] / R_{0}\left(R_{0}\right.$ denotes the resistivity at the coercive field, i.e., for a vanishing net magnetization) of the Ni film on (011) PMN-PT as a function of the magnetic field $\mathrm{H}$ applied together with the current $\mathrm{J}$ along the $\boldsymbol{y}$ (Figs. 1(a) and 1(b)) and $\boldsymbol{x}$ (Fig. 1(c)) sample axes under several electric fields applied to PMN-PT. Increasing the electric field from 0 to $1.6 \mathrm{kV} / \mathrm{cm}$, a slight increase of the saturation $\mathrm{MR}\left(\mathrm{MR}_{\max }\right)$ value from $1.68 \%$ to $1.81 \%$ is detected, as shown in Fig. 1(a). Further application of the $2.4 \mathrm{kV} / \mathrm{cm}$ field leads to the poling of PMN-PT, accompanied by its abrupt deformation and hence by a strong straininduced modification of the MR response of Ni film. A much higher $\mathrm{MR}_{\max }$ value of $2.34 \%$ and the significantly larger magnetic field, at which the MR saturates, $\left(\mathrm{H}_{\mathrm{s}}\right)$ are visible. An increase of the electric field to $4 \mathrm{kV} / \mathrm{cm}$ yields no further considerable change in the $\mathrm{MR}_{\max }$ but an increase in $\mathrm{H}_{\mathrm{s}}$, while subsequent decrease of the electric field down to $0 \mathrm{kV} / \mathrm{cm}$ leads to a decrease of $H_{s}$. Moreover, the $H_{s}$ value continues to decrease on subsequent application of negative voltage of $-2.4 \mathrm{kV} / \mathrm{cm}$, as shown in Fig. 1(b). This variation of the applied electric field is accompanied by a decrease of the $\mathrm{MR}_{\max }$ value from $2.27 \%$ to $1.96 \%$, implying an approximation to the coercive electric field, at which PMN-PT is near to the zero-polarization state and hence the Ni film on top of PMN-PT is under the unstrained condition, again showing approximate reversibility. However, applying then $-4 \mathrm{kV} / \mathrm{cm}$, we pole the piezosubstrate in opposite out-ofplain direction and obtain again the high $\mathrm{MR}_{\max }$ value of $2.2 \%$ and the large $\mathrm{H}_{\mathrm{s}}$, as expected from the symmetry of the poling.

Figure 1(c) shows the longitudinal MR of Ni film along the $\boldsymbol{x}$ direction under the same electric fields applied to PMN-PT (previously poled by $4 \mathrm{kV} / \mathrm{cm}$ ) as presented in

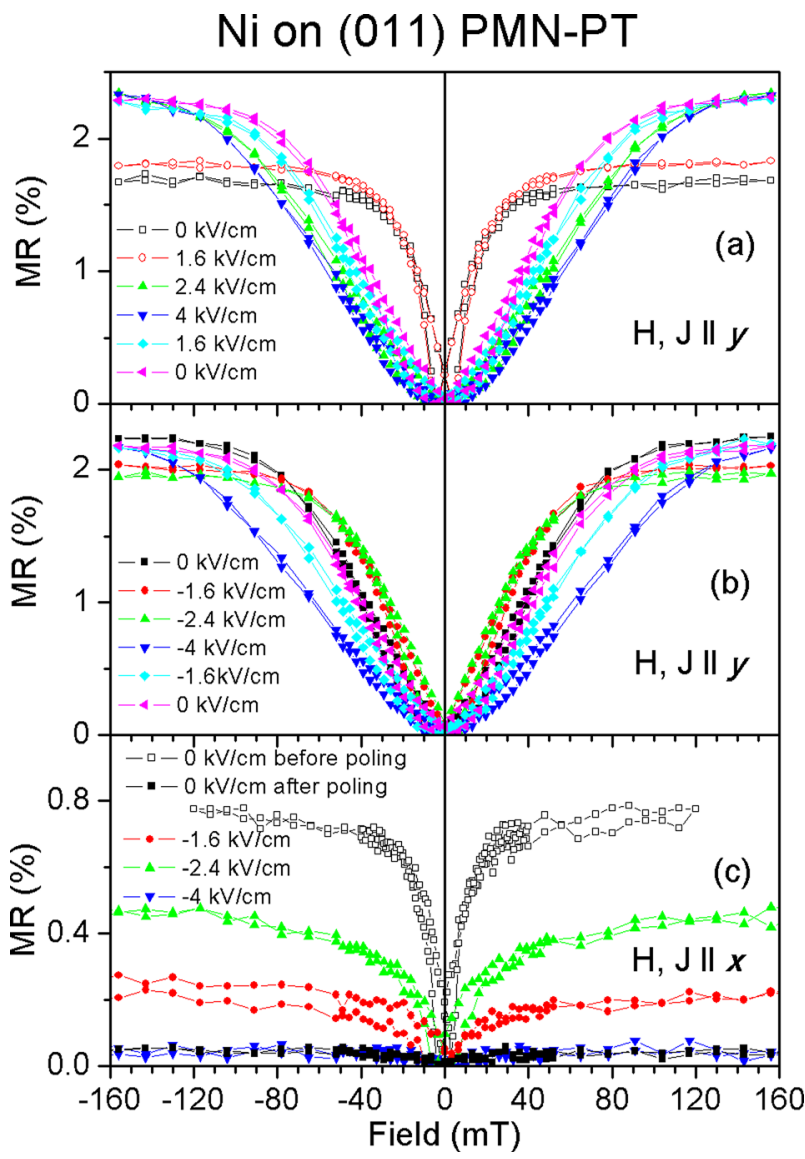

FIG. 1. Room-temperature magnetoresistance ratio $\Delta \mathrm{R} / \mathrm{R}_{0}$ of the $\mathrm{Ni}$ film on (011) PMN-PT as a function of magnetic field applied together with current along the $\boldsymbol{y}((\mathrm{a})$ and (b)) and $\boldsymbol{x}$ (c) in-plane directions, measured under positive electric fields varying as $0,1.6,2.4,4,1.6$, and $0 \mathrm{kV} / \mathrm{cm}$ (a) and under negative electric fields varying as $0,-1.6,-2.4,-4,-1.6$, and $0 \mathrm{kV} / \mathrm{cm}(\mathrm{b})$. In (c), we show the sequence for 0 (before poling), 0 (after poling with $4 \mathrm{kV} / \mathrm{cm}$ ), and then $-1.6,-2.4,-4 \mathrm{kV} / \mathrm{cm}$, applied to the piezosubstrate. The lines connecting points are guides to the eye.

Fig. 1(b) for the $y$ direction. For comparison, the MR response of the unpoled sample is depicted as well. We show that we can nearly completely suppress the MR response, poling PMN-PT, as the change in the MR response along $\boldsymbol{x}$ is reduced by a factor of $\sim 20$ from $0.78 \%$ to $0.04 \%$. Upon further application of $-1.6 \mathrm{kV} / \mathrm{cm}$ and $-2.4 \mathrm{kV} / \mathrm{cm}$, the $\mathrm{MR}_{\text {max }}$ value changes towards the unpoled one, reaching $0.22 \%$ and $0.46 \%$, respectively. However, subsequently applying $-4 \mathrm{kV} / \mathrm{cm}$, we suppress the $\mathrm{MR}_{\max }$ again showing that this effect is again reproducible and reversible. A factor of 4 suppression of the MR ratio and a decrease of the saturation field by application of the $4 \mathrm{kV} / \mathrm{cm}$ electric field were observed as well for $\mathrm{Ni}_{80} \mathrm{Fe}_{20} / \mathrm{PZN}-\mathrm{PT}$ heterostructures. ${ }^{14}$

In Figure 2, we analyze the variation of the $\mathrm{MR}_{\max }$ and $\mathrm{H}_{\mathrm{s}}$ values with the electric field. Results presented by solid symbols reveal a clear poling step around $2.4 \mathrm{kV} / \mathrm{cm}$ and a "butterfly" like loop with the coercive electric field of $-2.4 \mathrm{kV} / \mathrm{cm}$. Such a critical field value was also observed in the electric field dependence of the strain induced in the PMN-PT crystals of the same type and manufacturer. ${ }^{9}$ Moreover, the approximately linear variation of the $\mathrm{H}_{\mathrm{s}}$ with electric field decreasing from $4 \mathrm{kV} / \mathrm{cm}$ to 0 is in a qualitative agreement with the variation of the anisotropy field of $\mathrm{Ni}$ films on PMN-PT as a function of the electric field, deduced 


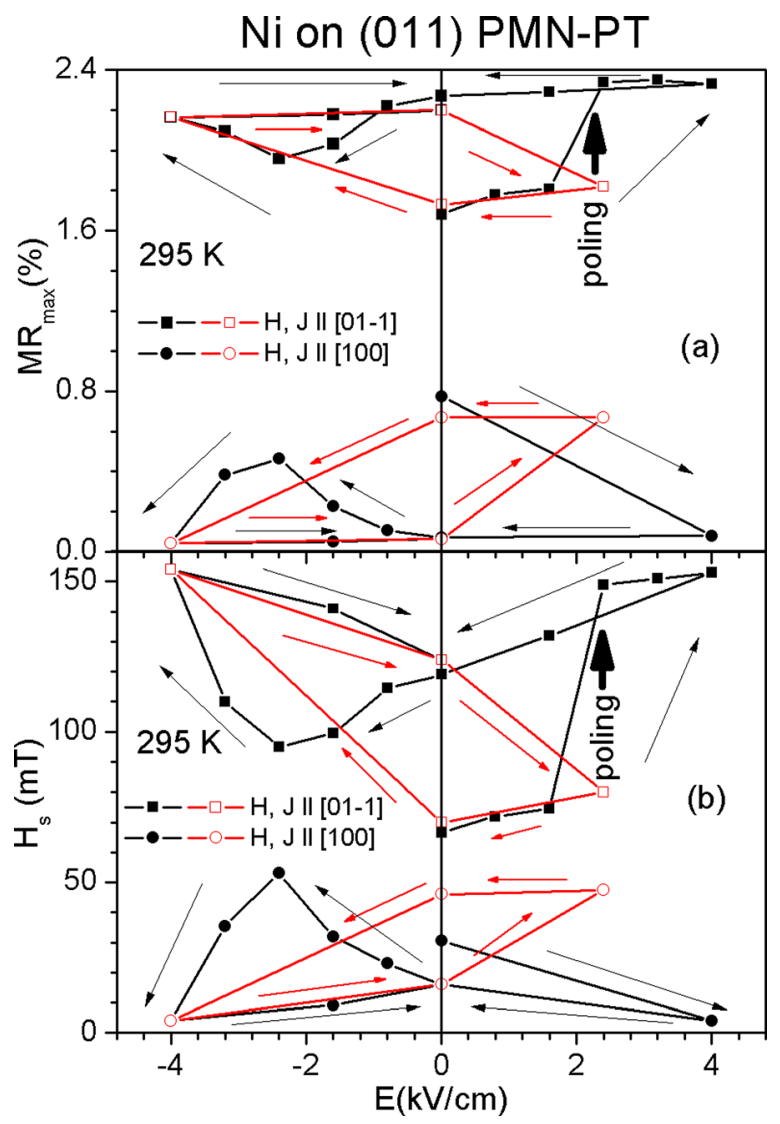

FIG. 2. Saturation longitudinal magnetoresistance value $\mathrm{MR}_{\max }$ (a) and field $\mathrm{H}_{\mathrm{s}}$ (b) of the Ni film on (011) PMN-PT with magnetic field and current along the $\boldsymbol{y}$ (squares) and $\boldsymbol{x}$ (circles) in-plane directions as a function of electric fields applied to the piezosubstrate in $0 \rightarrow 4 \rightarrow-4 \rightarrow 0 \mathrm{kV} / \mathrm{cm}$ sequence (solid symbols) and in $-4 \rightarrow 0 \rightarrow 2.4 \rightarrow 0 \mathrm{kV} / \mathrm{cm}$ sequence (open symbols). The lines connecting points are guides to the eye.

from MOKE magnetometry. ${ }^{6}$ Furthermore, a monotonous variation of $\mathrm{H}_{\mathrm{s}}$ with electric field was also observed for the MR response of $\mathrm{Ni}_{80} \mathrm{Co}_{20} / \mathrm{PZN}-\mathrm{PT}$ heterostructure. ${ }^{13}$ However, here we show by electrical read-out that we can change the magnetoresistance irreversibly and obtain different magnetoresistance values in a non-volatile manner at zero applied electric field, which has not been reported so far but is important for practical applications. Thus, knowing the value of the critical electric field, we have measured the MR of the Ni film on (011) PMN-PT at zero electric field after application of $-4 \mathrm{kV} / \mathrm{cm}$ (maximum residual strain in the poled state) and subsequent application of $2.4 \mathrm{kV} / \mathrm{cm}$ (minimum residual strain in the close to zero polarization state) to the piezosubstrate. As shown in Figure 3(a), the measurements were performed in both $\boldsymbol{y}$ and $\boldsymbol{x}$ current directions and both longitudinal and transverse geometries and compared to the results obtained in the unpoled state. Both directions are found to exhibit a typical anisotropic MR (AMR) curve. The resistivity of the $\mathrm{Ni}$ film in the longitudinal (transverse) direction shows minima (maxima) near the coercive field of $\sim 4 \mathrm{mT}$, increases (decreases), and saturates to a constant value $\mathrm{MR}_{\max }$ above the saturation field $\mathrm{H}_{\mathrm{s}}$. However, the parameters of the MR response $\left(\mathrm{MR}_{\max }\right.$ and $\left.\mathrm{H}_{\mathrm{s}}\right)$ depend sensitively on the magnetic field direction. When the magnetic field is along $\boldsymbol{y}$, the absolute values of $\mathrm{MR}_{\max }$ and $\mathrm{H}_{\mathrm{s}}$ are higher than that for magnetic field along $\boldsymbol{x}$. Moreover, these

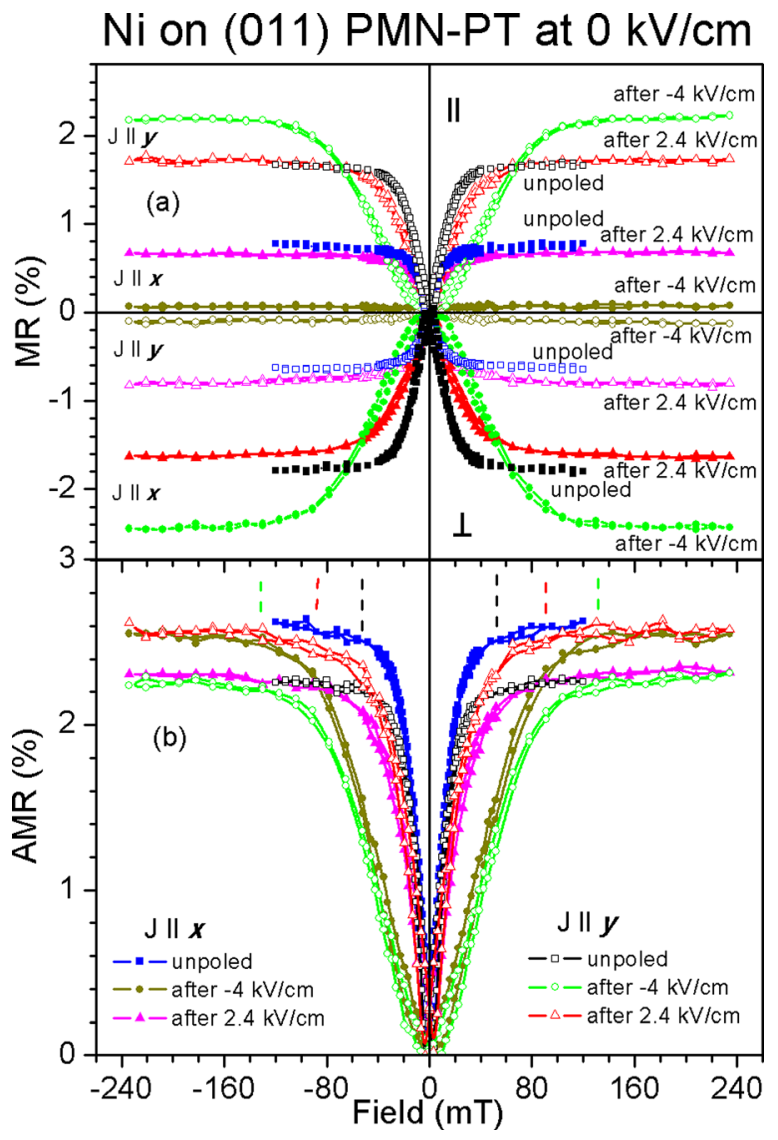

FIG. 3. Room-temperature magnetoresistance ratio $\Delta \mathrm{R} / \mathrm{R}_{0}$ in longitudinal $(\|)$ and transverse $(\perp)$ geometries (a) and anisotropic magnetoresistance (b) of the Ni film on (011) PMN-PT in unpoled state (squares), after poling with application of the $-4 \mathrm{kV} / \mathrm{cm}$ electric field (circles), and after subsequent application of the $2.4 \mathrm{kV} / \mathrm{cm}$ electric field (triangles) as function of magnetic field, measured with the current along the $\boldsymbol{x}$ (solid symbols) and $\boldsymbol{y}$ (open symbols) in-plane directions. Dashed lines mark the anisotropy fields. The lines connecting points are guides to the eye.

values increase after the application of the $-4 \mathrm{kV} / \mathrm{cm}$ electric field and decrease close to the initial value in the unpoled state after further application of the $2.4 \mathrm{kV} / \mathrm{cm}$ electric field for both $\boldsymbol{y}$ and $\boldsymbol{x}$ currents. In contrast, $\mathrm{MR}_{\max }$ diminishes upon poling and recovers at de-poling for the magnetic field aligned along $\boldsymbol{x}$. Such asymmetric hysteretic behavior of the MR parameters as a function of electric field is presented by the open symbols in Figure 2 for the longitudinal MR.

Using the results plotted in Figure 3(a), the anisotropic magnetoresistance was defined as $A M R=\left[R_{\text {long }}-R_{\text {trans }}\right] /$ $\left[R_{\text {long }} / 3+2 \cdot R_{\text {trans }} / 3\right]$, where $R_{\text {long }}$ and $R_{\text {trans }}$ are the resistances for magnetic field applied parallel and perpendicular to the current direction, respectively. Figure 3(b) shows the room-temperature AMR ratio of the Ni film on (011) PMNPT in unpoled state, after poling by the application of $-4 \mathrm{kV} / \mathrm{cm}$ and after subsequent application of $2.4 \mathrm{kV} / \mathrm{cm}$ for the currents along $\boldsymbol{x}$ and $\boldsymbol{y}$. For all the cases, the maximum AMR values are similar, varying between $2.31 \%$ and $2.62 \%$, and being just slightly smaller than $\sim 3 \%$ observed for a $\mathrm{Ni}_{77} \mathrm{Fe}_{23} / \mathrm{PMN}-\mathrm{PT}$ heterostructure. ${ }^{17}$ The effect of the electric field pulse application is mainly reflected in the modification of the anisotropy field $\mathrm{H}_{\mathrm{a}}$ (marked in Fig. 3(b) by dashed lines) when the magnetization is aligned with the applied magnetic field. Upon poling, $\mathrm{H}_{\mathrm{a}}$ value increases from 
$\sim 54 \mathrm{mT}$ to $\sim 132 \mathrm{mT}$ as observed for both $\boldsymbol{x}$ and $\boldsymbol{y}$ current directions. Further application of the $2.4 \mathrm{kV} / \mathrm{cm}$ pulse leads to the decrease of $\mathrm{H}_{\mathrm{a}}$ to $\sim 87 \mathrm{mT}$.

To understand the results, we compare the observed field-dependence with magnetometry. We find that the variation of $\mathrm{H}_{\mathrm{a}}$ is confirmed by the magnetization loop measurements of Ni films on PMN-PT as presented in Figure 4. In the unpoled state (Fig. 4(a)), the magnetization loop for the $x$ magnetic field direction is just slightly smaller than that for the $\boldsymbol{y}$ direction and both loops merge at magnetic field as low as $\sim 60 \mathrm{mT}$ that is near the $\mathrm{H}_{\mathrm{a}}$ value deduced from the transport measurements. After application of the $-4 \mathrm{kV} / \mathrm{cm}$ electric field (Fig. 4(b)), the loops become distinctly different. In the $\boldsymbol{y}$ magnetic field direction, it is more square-shaped, while along $\boldsymbol{x}$ the loop is slanted. So the loops merge at much larger $\mathrm{H}_{\mathrm{a}}$ value close to $130 \mathrm{mT}$, in agreement with the AMR analysis. Finally, further application of a $2.4 \mathrm{kV} / \mathrm{cm}$ electric field (Fig. 4(c)) leads to a partial recovery of the initial magnetic state with $\mathrm{H}_{\mathrm{a}}$ of $\sim 90 \mathrm{mT}$. This means that we have achieved a non-volatile permanent magnetization change that is reflected in the magnetotransport properties.

At the same time, the coercive magnetic field $\left(\mathrm{H}_{\mathrm{c}}\right)$ values, which are deduced from the magnified loops as shown in Figure 5, reveal a good correlation with the $\mathrm{H}_{\mathrm{s}}$ parameter of the longitudinal MR, presented in Figure 2(b). After poling, $\mathrm{H}_{\mathrm{c}}$ increases from $4.1 \mathrm{mT}$ to $8.8 \mathrm{mT}\left(\mathrm{H}_{\mathrm{s}}\right.$ increases from $66.5 \mathrm{mT}$ to $124 \mathrm{mT}$ ) along $\boldsymbol{y}$ and slightly

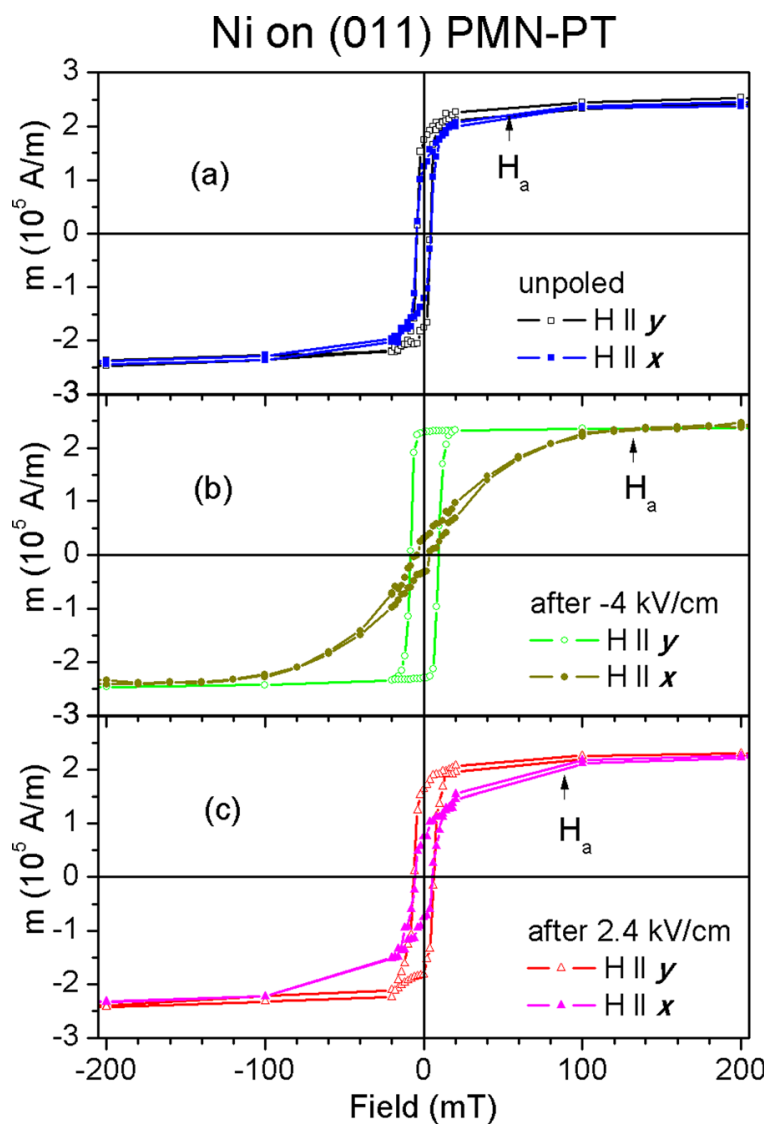

FIG. 4. Room-temperature magnetization loops of the $\mathrm{Ni}$ film on (011) PMN-PT, measured by the SQUID along the $\boldsymbol{x}$ (solid symbols) and $\boldsymbol{y}$ (open symbols) directions in unpoled state (a), after poling with application of the $-4 \mathrm{kV} / \mathrm{cm}$ electric field (b), and after subsequent application of the $2.4 \mathrm{kV} /$ $\mathrm{cm}$ electric field (c). The lines connecting points are guides to the eye.

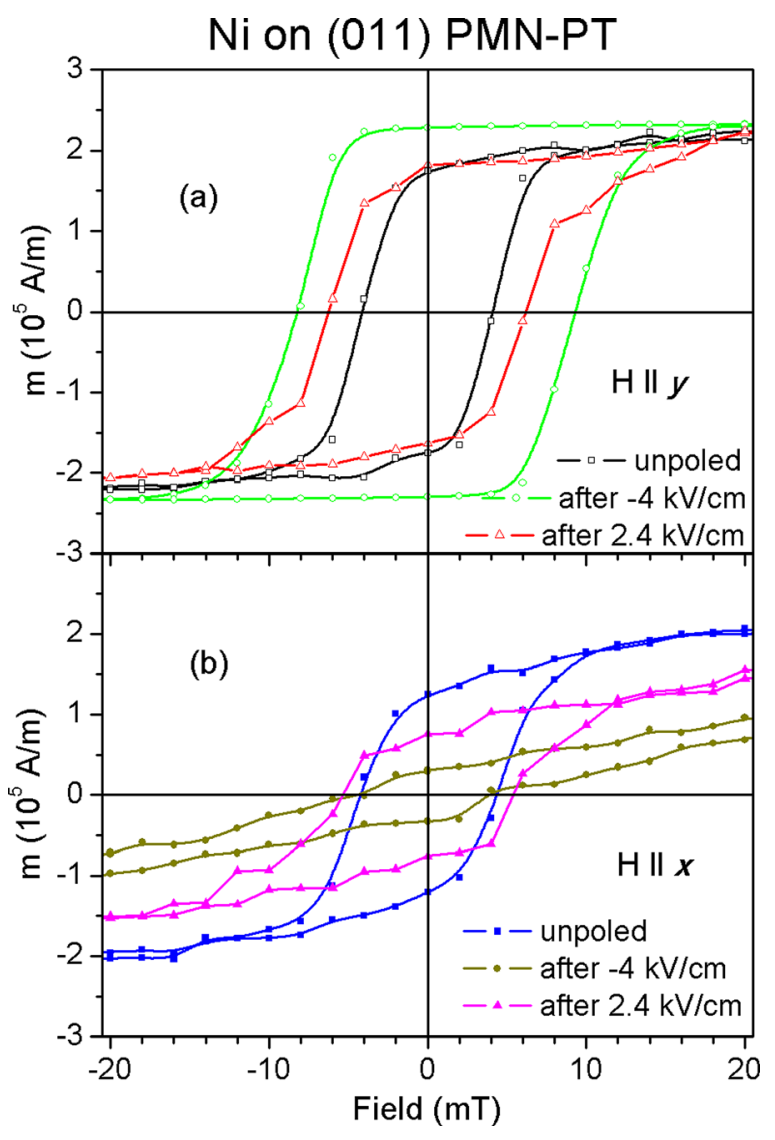

FIG. 5. Magnified room-temperature magnetization loops of the Ni film on (011) PMN-PT, measured by the SQUID along the $\boldsymbol{y}$ (a, open symbols) and $\boldsymbol{x}$ (b, solid symbols) directions unpoled state (squares), after poling with application of the $-4 \mathrm{kV} / \mathrm{cm}$ electric field (circles), and after subsequent application of the $2.4 \mathrm{kV} / \mathrm{cm}$ electric field (triangles). The lines connecting points are guides to the eye.

decreases from $4.3 \mathrm{mT}$ to $4.2 \mathrm{mT}\left(\mathrm{H}_{\mathrm{s}}\right.$ decreases from $30.5 \mathrm{mT}$ to $16 \mathrm{mT}$ ) along $\boldsymbol{x}$. After the application of $2.4 \mathrm{kV} / \mathrm{cm}$ subsequent to $-4 \mathrm{kV} / \mathrm{cm}, \mathrm{H}_{\mathrm{c}}$ decreases to $6.2 \mathrm{mT}$ $\left(\mathrm{H}_{\mathrm{s}}\right.$ decreases to $\left.70 \mathrm{mT}\right)$ in the $\boldsymbol{y}$ direction and increases to $5.4 \mathrm{mT}\left(\mathrm{H}_{\mathrm{s}}\right.$ decreases to $\left.46 \mathrm{mT}\right)$ in the $\boldsymbol{x}$ direction. In agreement with our results, a coercivity of $\sim 3.5 \mathrm{mT}$ was reported for the as-deposited Ni film on $\mathrm{LiNbO}_{3}$ substrate along the $\boldsymbol{y}$ direction, while the electric poling induced the remanent strain change, yielding an $\mathrm{H}_{\mathrm{c}}$ of $\sim 8 \mathrm{mT}$. $^{6}$

Instead of the previously used semi-quantitative MOKE magnetometry, ${ }^{7,8}$ here we use a fully quantitative SQUID technique to determine not only the critical magnetic field values but also a saturation magnetization and anisotropy constants quantitatively. Thus, determining the saturation magnetization value as $255 \pm 15 \mathrm{kA} / \mathrm{m}$ and taking the $\mathrm{H}_{\mathrm{a}}$ values deduced from MR study, the absolute value of an anisotropy constant, estimated as their product divided by $2,{ }^{23,24}$ is found to be $\sim 7 \mathrm{~kJ} / \mathrm{m}^{3}$ for unpoled state, $\sim 17 \mathrm{~kJ} / \mathrm{m}^{3}$ after poling, and $\sim 11 \mathrm{~kJ} / \mathrm{m}^{3}$ after de-poling pulse. Such variation of the anisotropy constant is in good agreement with the magnetoelastic anisotropy up to $16 \mathrm{~kJ} / \mathrm{m}^{3}$ induced by poling the (011) PMN-PT. ${ }^{9}$

In conclusion, we have demonstrated a very large effect of the poling of (011) PMN-PT piezosubstrates on the magnetic anisotropy and magnetotransport properties of $\mathrm{Ni}$ films at room temperature. The anisotropy field and thus 
anisotropy constant are increased $\sim 2.5$ times. At the same time, the longitudinal magnetoresistance is reduced by a factor of $\sim 20$ for the $x$ current direction, indicating an induced hard axis, and increased for the $\boldsymbol{y}$ current direction, indicating an easy axis. The modification of the MR response by the applied electric field was attributed to a change in the straininduced anisotropy of the films induced by the PMN-PT substrate with piezocoefficients of different signs in orthogonal in-plane $\boldsymbol{x}$ and $\boldsymbol{y}$ directions. Moreover, we have shown a reversible and non-volatile variation of the magnetic anisotropy in the Ni films on (011) PMN-PT using voltage pulses in the non-linear regime of the piezoelectric PMN-PT. This allows us to switch the magnetotransport and magnetic behavior of the Ni films between the poled high strain state and near-zero-polarization low strain state. The magnetic anisotropy induced by poling and switching of the magnetotransport and magnetic properties provides a promising approach for magnetic field sensor and memory applications.

This work was funded by the EU's 7th Framework Program IFOX (NMP3-LA-2010 246102), the Graduate School of Excellence MAINZ (GSC 266 Mainz), the German Science Foundation (DFG), and the ERC (2007-Stg 208162). A. Tkach also acknowledges funds by FEDER through Programa Operacional Factores de CompetitividadeCOMPETE, and national funds through FCT-Fundação para a Ciência e Tecnologia within CICECO projectFCOMP-01-0124-FEDER-037271 (FCT Ref. PEst-C/CTM/ LA0011/2013) and independent researcher Grant No. IF/ 00602/2013.

${ }^{1}$ J. Ma, J. Hu, Z. Li, and C.-W. Na, Adv. Mater. 23, 1062 (2011).

${ }^{2}$ C. A. F. Vaz, J. Phys.: Condens. Matter 24, 333201 (2012).

${ }^{3}$ J. T. Heron, D. G. Schlom, and R. Ramesh, Appl. Phys. Rev. 1, 021303 (2014).
${ }^{4}$ M. Liu and N. X. Sun, Philos. Trans. R. Soc., A 372, 20120439 (2014).

${ }^{5}$ M. Liu, O. Obi, J. Lou, Y. Chen, Z. Cai, S. Stoute, M. Espanol, M. Lew, X. Situ, K. S. Ziemer, V. G. Harris, and N. X. Sun, Adv. Funct. Mater. 19, 1826 (2009)

${ }^{6}$ T. Wu, A. Bur, J. L. Hockel, K. Wong, T.-K. Chung, and G. P. Carman, IEEE Magn. Lett. 2, 6000104 (2011).

${ }^{7}$ T. Wu, A. Bur, K. Wong, J. L. Hockel, C.-J. Hsu, H. K. D. Kim, K. L. Wang, and G. P. Carman, J. Appl. Phys. 109, 07D732 (2011).

${ }^{8}$ T. Wu, A. Bur, P. Zhao, K. P. Mohanchandra, K. Wong, K. L. Wang, C. S. Lynch, and G. P. Carman, Appl. Phys. Lett. 98, 012504 (2011).

${ }^{9}$ J. L. Hockel, A. Bur, T. Wu, K. P. Wetzlar, and G. P. Carman, Appl. Phys. Lett. 100, 022401 (2012).

${ }^{10}$ H. K. D. Kim, L. T. Schelhas, S. Keller, J. L. Hockel, S. H. Tolbert, and G. P. Carman, Nano Lett. 13, 884 (2013).

${ }^{11}$ S. Finizio, M. Foerster, M. Buzzi, B. Krüger, M. Jourdan, C. A. F. Vaz, J. Hockel, T. Miyawaki, A. Tkach, S. Valencia, F. Kronast, G. P. Carman, F. Nolting, and M. Kläui, Phys. Rev. Appl. 1, 021001 (2014).

${ }^{12}$ V. Laukhin, V. Skumryev, X. Marti, D. Hrabovsky, F. Sanchez, M. V. Garcia-Cuenca, C. Ferrater, M. Varela, U. Luders, J. F. Bobo, and J. Fontcuberta, Phys. Rev. Lett. 97, 227201 (2006).

${ }^{13}$ M. Liu, S. Li, O. Obi, J. Lou, S. Rand, and N. X. Sun, Appl. Phys. Lett. 98, 222509 (2011).

${ }^{14}$ M. Liu, O. Obi, J. Lou, S. Li, X. Xing, G. Yang, and N. X. Sun, J. Appl. Phys. 109, 07D913 (2011).

${ }^{15}$ A. Brandlmaier, S. Geprags, G. Woltersdorf, R. Gross, and S. T. B. Goennenwein, J. Appl. Phys. 110, 043913 (2011).

${ }^{16} \mathrm{M}$. Liu, J. Hoffman, J. Wang, J. Zhang, B. Nelson-Cheeseman, and A. Bhattacharya, Sci. Rep. 3, 1876 (2013).

${ }^{17}$ Y. Gao, J. Hu, L. Shu, and C. W. Nan, Appl. Phys. Lett. 104, 142908 (2014).

${ }^{18}$ D. Preziosi, I. Fina, E. Pippel, D. Hesse, X. Marti, F. Bern, M. Ziese, and M. Alexe, Phys. Rev. B 90, 125155 (2014).

${ }^{19}$ J. H. Lee, I. Fina, X. Marti, Y. H. Kim, D. Hesse, and M. Alexe, Adv. Mater. 26, 7078 (2014).

${ }^{20}$ A. Tkach, M. Baghaie Yazdi, M. Foerster, F. Büttner, M. Vafaee, M. Fries, and M. Kläui, Phys. Rev. B 91, 024405 (2015).

${ }^{21}$ J. Allibe, S. Fusil, K. Bouzehouane, C. Daumont, D. Sando, E. Jacquet, C. Deranlot, M. Bibes, and A. Barthelemy, Nano Lett. 12, 1141 (2012).

${ }^{22}$ H. Kojima, T. Naito, H. Muraoka, E. Wada, I. Suzuki, Y. Shirahata, M. Itoh, and T. Taniyama, J. Appl. Phys. 113, 17C713 (2013).

${ }^{23}$ E. C. Stoner and E. P. Wohlfarth, Philos. Trans. R. Soc., A 240, 599 (1948).

${ }^{24}$ M. Ziese, I. Vrejoiu, and D. Hesse, Phys. Rev. B 81, 184418 (2010). 